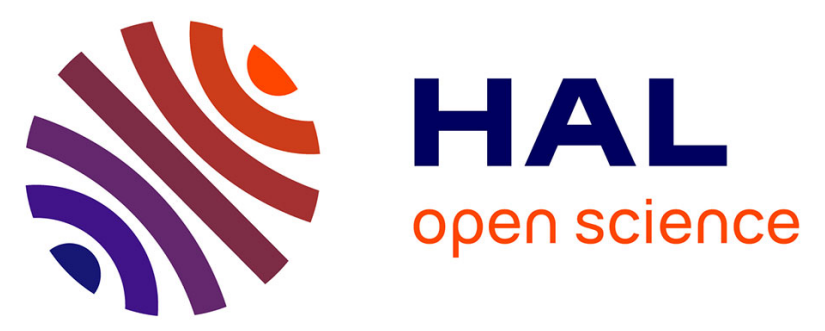

\title{
A substructured version of the variational theory of complex rays dedicated to the calculation of assemblies with dissipative joints in the medium-frequency range Olivier Dorival, Philippe Rouch, Olivier Allix
}

\section{- To cite this version:}

Olivier Dorival, Philippe Rouch, Olivier Allix. A substructured version of the variational theory of complex rays dedicated to the calculation of assemblies with dissipative joints in the medium-frequency range. Engineering Computations, 2006, 23 (7-8), pp.729-748. 10.1108/02644400610689875 . hal01023404

\author{
HAL Id: hal-01023404 \\ https://hal.science/hal-01023404
}

Submitted on 12 Jul 2014

HAL is a multi-disciplinary open access archive for the deposit and dissemination of scientific research documents, whether they are published or not. The documents may come from teaching and research institutions in France or abroad, or from public or private research centers.
L'archive ouverte pluridisciplinaire HAL, est destinée au dépôt et à la diffusion de documents scientifiques de niveau recherche, publiés ou non, émanant des établissements d'enseignement et de recherche français ou étrangers, des laboratoires publics ou privés. 


\title{
A SUBSTRUCTURED VERSION OF THE VARIATIONAL THEORY OF COMPLEX RAYS DEDICATED TO THE CALCULATION OF ASSEMBLIES WITH DISSIPATIVE JOINTS IN THE MEDIUM-FREQUENCY RANGE
}

\author{
O. Dorival, P. Rouch, O. Allix \\ LMT Cachan - ENS de Cachan/Paris VI/CNRS \\ 61, av. du Pt Wilson - 94235 Cachan Cedex \\ dorival@lmt.ens-cachan.fr \\ rouch@lmt.ens-cachan.fr \\ allix@lmt.ens-cachan.fr
}

\begin{abstract}
Keywords: Joint Modeling, Medium Frequency, Damping, VTCR, Substructuration, Variational Approach

This paper focuses on efficient techniques for the analysis of complex structures in the mediumfrequency range taking into account elaborate joint behavior. The accurate prediction of the amplitude of the response in this frequency range requires sophisticated interface models. Indeed, experiments on generic assemblies clearly showed that joint stiffness and damping must be taken into account. We deal with these problems through the use of a dedicated approach, the Variational Theory of Complex Rays (VTCR). This method can be viewed as a means of expressing the power balance at the different boundaries between substructures (hence the importance of interface modeling) using two-scale shape functions which verify the dynamic equation and the constitutive relation, which leads to very low computational costs. Despite the fact that the classical VTCR formulation is entirely dedicated to such calculations, it has shortcomings. Here, we introduce an original substructured version of the VTCR which enables the equations of the joints to be separated from those of the substructures in order to improve the flexibility of the behavior at the interfaces.
\end{abstract}

\section{Introduction}

The prediction of the response of a complex structure in the medium-frequency (MF) range presents two major difficulties. The first difficulty is that the solution of a problem involves small-wavelength phenomena. Therefore, using the finite element method (FEM), the calculation of this solution leads to a huge number of degrees of freedom. Indeed, at least seven elements per wavelength are necessary to represent oscillating solutions properly. Moreover, since $k^{3} h^{2}$ must remain constant (Deraemaeker, et al. 1999), the cost of FE analysis increases with the frequency. The second difficulty is that due to the high modal density in the MF domain the response is governed mainly by the level and the nature of the dissipative phenomena, whose mechanisms are very difficult to describe, model and measure. In the case of thin structures - such as car chassis, satellites or submarines -, damping is localized mainly in the joints between substructures, which can be of different types.

Many research works have been devoted to answering the first point. Some aim at extending the classical low-frequency approaches (FEM) to the medium-frequency range 
(Farhat, et al. 2001, Farhat, et al. 2003, Harari \& Haham 1998). Other approaches, such as Statistical Energy Analysis (SEA) (Lyon \& Maidanick 1967), try to relax some of the assumptions associated with high-frequency methods in order to become applicable to the MF domain.

In this study, we use the Variational Theory of Complex Rays (VTCR) (Ladevèze, et al. 2001), which deals with the first difficulty by using two-scale shape functions which verify the dynamic equations and the constitutive law of the substructures. This leads to a small, frequency-independent linear system (Rouch \& Ladevèze 2003, Ladevèze \& Riou 2005). We focus particularly on the second difficulty.

Noting that one of the specificities of the VTCR is its variational formulation of all the boundary conditions between substructures, we concentrate on the modeling of joints between substructures: this feature allows us to develop the equations of complex interfaces involving a dissipative part easily, which is necessary to obtain accurate predictions of the behavior of real structures.

To improve this process, we derive a substructured version of the VTCR which allows us to treat interfaces with greater flexibility. Indeed, in the classical version of the VTCR, all the interface conditions of the entire structure are coupled, which can be exceedingly involved to improve only some joint equations, for example when updating the transmission conditions of the structure using experimental data or when analyzing multiresolution problems. This substructured reformulation is based on a condensation approach which consists in separating the properly modeled substructures and boundaries from the more inaccurate complex joints. After the introduction of interface degrees of freedom, one ends up with a constrained minimization problem.

Finally, in order to predict the behavior of real structures accurately, we introduce models of complex joints involving a dissipative part. These models were implemented into the VTCR. We present applications concerning 3D plate assemblies connected by heterogeneous joints with distributed parameters (such as mass, stiffness and damping) which can be identified on generic assemblies.

\section{A substructured version of the VTCR}

\subsection{Introduction}

The classical version of the VTCR (Ladevèze et al. 2001), which will not be detailed in this paper, is entirely dedicated to the modeling of joints, since it is based on the decomposition of the structure into simple substructures, which involves transmission conditions. However, this formulation treats all the boundaries and interfaces of the structure globally, which makes it cumbersome for studying the vibrations of the same structure with different joint equations if, for example, one wants to model the interfaces using stochastic interface parameters to describe the dispersion due to the production process. Therefore, after introducing the reference problem, we derive a substructured version of the VTCR.

Many substructuration methods have appeared from the beginning of numerical methods. The first studies were based on modal analysis of each substructures (see the Com- 
ponent Modal Synthesis (CMS) (Hurty 1965)). The Craig-Bampton approach proposes to compute substructures modes with clamped interfaces (Craig \& Bampton 1968). In the McNeal method, the substructures modes are computed with free interface conditions (McNeal 1971). Farhat and Géradin proposed a method allowing non compatible interfaces (Farhat \& Géradin 1994).

Other domain decomposition methods are not based on modal analysis, but introduce various interface quantities : the Balancing Domain Decomposition method (Roux 1990, Mandel 1993) uses primal quantities, whereas the FETI method (Farhat \& Roux 1991, Farhat, et al. 1994, Farhat \& Mandel 1998) uses dual interface quantities. Hybrid methods like the LATIN approach (Ladevèze 1998) propose not to favour dual or primal quantities.

\subsection{The reference problem}

Here, for the sake of simplicity, the problem will be formulated for an assembly of only two substructures, but this can be easily generalized to an assembly of $n$ substructures. As an illustration, let us consider two thin, homogeneous, isotropic and elastic KirchhoffLove plates and study the steady-state vibrations of this assembly. Classically, all quantities are defined in the complex domain and depend on the space quantities alone: the complex quantity $Q_{(\underline{X})}$ is associated with $Q_{(\underline{X}, t)}$ by the relation $Q_{(\underline{X}, t)}=Q_{(\underline{X})} \cdot \exp (i \omega t)$, where $\omega$ is the fixed angular frequency.

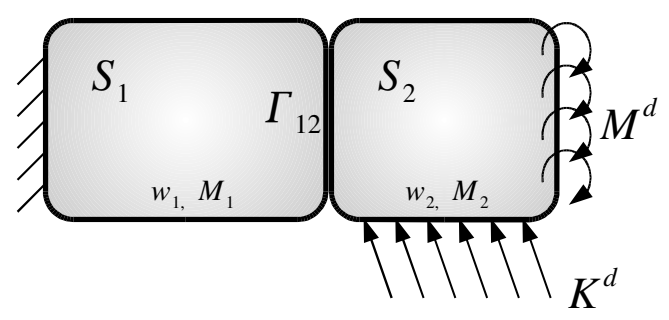

Fig. 1. The reference problem

Let $S_{1}$ and $S_{2}$ be two plates, $\partial S_{1}$ and $\partial S_{2}$ their respective boundaries solicited harmonically at a fixed angular frequency $\omega$, and $\Gamma_{12}$ the interface between $S_{1}$ and $S_{2}$, which, for clarity, will first be considered to be perfectly rigid. The boundary conditions are the following, with $l=1,2$ : prescribed out-of-plane displacement $w_{l}^{d}$ on a part $\partial_{w^{d}} S_{l}$ of $\partial S_{l}$, prescribed slope $w_{\underline{n}, l}^{d}$ on $\partial_{w_{n}^{d}} S_{l}$, prescribed bending moment $M_{l}^{d}$ on $\partial_{M^{d}} S_{l}$ and, finally, prescribed Kirchhoff shear $\bar{K}_{l}^{d}$ on $\partial_{K^{d}} S_{l}$ (see Fig. 1). The quantities of interest are the displacements $w_{l}$ and the moments $\mathbf{M}_{l}$.

For $S_{l}$, let us introduce the admissibility space $\mathcal{S}_{a d}^{l}$ of the displacement-moment pairs $s_{l}=\left(w_{l}, \mathbf{M}_{l}\right)$ defined on $S_{l}$ such that: 


$$
s_{l} \in \mathcal{S}_{a d}^{l} \Leftrightarrow\left\{\begin{array}{c}
w_{l} \in \mathcal{U} \quad\left(\text { the set of the finite-energy displacement fields } H_{(S)}^{2}\right) \\
\mathbf{M}_{l} \in \mathcal{S} \quad\left(\text { the set of the finite-energy moment fields }\left[L_{(S)}^{2}\right]^{3}\right) \\
\Delta \Delta w_{l}-k_{l}^{4} w_{l}=0 \quad \text { on } S_{l} \\
\mathbf{M}_{l}=\frac{2 h_{l}^{3}}{3}\left(1+i \eta_{l}\right) \mathbf{K}_{l, P S} \mathcal{X}\left(w_{l}\right) \\
\text { for } \quad l=1,2
\end{array}\right.
$$

with $\quad k_{l}^{4}=\frac{3 \rho_{l} \omega^{2}\left(1-\nu_{l}^{2}\right)}{\left(1+i \eta_{l}\right) E_{l} h_{l}^{2}}$

where $k_{l}$ is the wave number and $\rho_{l}, \nu_{l}, \eta_{l}, E_{l}$ and $2 h_{l}$ denote respectively the density, Poisson's ratio, structural damping coefficient, Young's modulus and thickness of Plate $S_{l}$. $\mathbf{K}_{l, P S}$ is Hooke's tensor for plane stress and $\mathcal{X}$ is the curvature operator. The fields of $\mathcal{S}_{a d}^{l}$ are admissible in the sense that they verify both the local equilibrium and the constitutive relation exactly. The reference problem is:

Problem 1. Find $s_{l}$ such that: $s_{l} \in \mathcal{S}_{a d}^{l}$

$$
\left\{\begin{array}{ccc|ccc}
w_{l}=w_{l}^{d} & \text { on } & \partial_{w^{d}} S_{l} & w_{1}=w_{2} & \text { on } & \Gamma_{12} \\
w_{l, \underline{n}_{l}}=w_{\underline{n}_{l}}^{d} & \text { on } & \partial_{w_{\underline{n}}^{d}} S_{l} & w_{1, \underline{n}_{1}}=w_{2, \underline{n}_{2}} & \text { on } & \Gamma_{12} \\
M_{l}=M_{l}^{d} \quad \text { on } & \partial_{M^{d}} S_{l} & M_{1}+M_{2}=0 & \text { on } & \Gamma_{12} \\
K_{l}=K_{l}^{d} & \text { on } & \partial_{K^{d}} S_{l} & K_{1}+K_{2}=0 & \text { on } & \Gamma_{12} \\
\text { for } & \quad l=1,2 & & & &
\end{array}\right.
$$

with $\quad M_{l}=\underline{n}_{l} \mathbf{M}_{l} \underline{n}_{l} \quad$ and $\quad K_{l}=\underline{n}_{l} \underline{\operatorname{div}}\left[\mathbf{M}_{l}\right]+\left(\underline{t}_{l} \mathbf{M}_{l} \underline{n}_{l}\right)_{, t_{l}}$

The reference problem has one solution if (Ladevèze et al. 2001):

- Hooke's tensors are positive definite,

- $\eta_{l}>0$.

\subsection{A substructured version of the VTCR}

Let us introduce a substructured version of the VTCR which consists in separating the properly modeled substructures from the less accurate complex joints. The cornerstone of this new formulation is the introduction of interface quantities, which we denote $\overline{\boldsymbol{}}$. Then, the interface equations can be solved by minimizing a functional under a set of constraints expressing the compatibility between substructures and interfaces in the form of classical VTCR problems, which will be briefly reminded. In doing this, we ensure that the solution within the substructures is exact: the only error, which is perfectly acceptable, is in the VTCR discretization of the substructures (anterior studies(Rouch \& Ladevèze 2003) have focused on the influence of the VTCR discretization and it will not be the subject of the 
present paper). Besides, the joints are dealt with less accurately through the minimization problem. This reformulation gives us greater flexibility to modify the interface equations: the substructures' minimization problem with constraints is built once and for all, and the calculation with a different set of joints requires changes only in the minimized interface residual. In future works, we will take advantage of this new formulation to calculate stochastic joint behavior, to deal with multiresolution problems or to identify joint parameters. In this paper, we concentrate on the presentation of this new formulation.

\subsubsection{Formulation of the continuous substructured VTCR}

This reformulation is based on the separation of the equations of the joints through the introduction of interface quantities. For clarity, we present only the separation of the interface's bending behavior. Considering the interface $\Gamma_{12}$, we introduce the interface quantities $\left(\overline{\bar{w}}_{1, \underline{n}_{1}}, \overline{\bar{M}}_{1}\right)$ and $\left(\overline{\bar{w}}_{2, \underline{n}_{2}}, \overline{\bar{M}}_{2}\right)$ of Substructures 1 and 2 respectively. Each interface quantity $\left(\overline{\bar{w}}_{l}, \underline{\underline{n}}_{l}, \overline{\bar{M}}_{l}\right)$ must be equal to the corresponding substructure quantity $\left(w_{l, \underline{n}_{l}}, M_{l}\right)$ (at least in the continuous case). The interface, which can be viewed as a real entity, has its own behavior described by an equilibrium equation and a constitutive law involving the interface quantities $\left(\overline{\bar{w}}_{1, \underline{n}_{1}}, \overline{\bar{M}}_{1}\right)$ and $\left(\overline{\bar{w}}_{2, \underline{n}_{2}}, \overline{\bar{M}}_{2}\right)$. Thus, the basic problem is divided into two subproblems (see Fig. 2): Substructure $l$ must verify a substructure/interface compatibility problem while the interface quantities must minimize a $L^{2}$ residual of joint equations.

\subsubsection{The substructure/interface compatibility problem}

Among several possible options, we chose a condensation approach with which prescribed interface quantities $\overline{\bar{M}}_{l}$ :
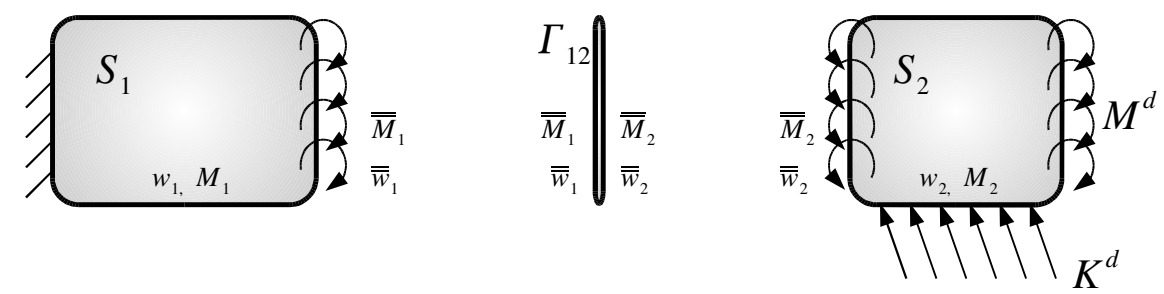

Fig. 2. The VTCR problem with substructuration 
Substructure l/interface compatibility problem:

- dynamic equation

- constitutive relation

- boundary conditions: prescribed slope:

$$
\begin{aligned}
& w_{\underline{n}, l}^{d}=w_{\underline{n}, l}^{d} \quad \text { on } \partial_{w_{\underline{n}}^{d}} S_{l} \\
& \begin{array}{lll}
\text { prescribed bending moments: } & M_{l}=M_{l}^{d} & \text { on } \partial_{M}^{d^{\underline{n}}} S_{l} \\
\text { prescribed interface quantities: } & M_{l}=\overline{\bar{M}}_{l} & \text { on } \Gamma_{12}
\end{array} \\
& w_{\underline{n}, l}=\overline{\bar{w}}_{\underline{n}, l} \quad \text { on } \Gamma_{12}
\end{aligned}
$$

This problem is solved through the use of the VTCR, which we are about to introduce. The VTCR is primarily a global formulation of the boundary conditions and transmission conditions in terms of both displacements and moments:

Problem $2_{l}$. Find $s_{l}$ in $S_{a d}^{l}$ such that:

$$
\left\{\begin{array}{c}
A_{\partial S_{l}}\left(\delta s_{l}, s_{l}\right)-L_{\partial S}\left(\delta s_{l}\right)+C_{\Gamma_{l}}\left(\delta s_{l}, s_{l}, \bar{s}_{l}\right)=0 \\
\forall \delta s_{l} \in \mathcal{S}_{a d}^{l} \text { for } l=1,2
\end{array}\right.
$$

where:

$$
\begin{aligned}
A_{\partial S_{l}}\left(\delta s_{l}, s_{l}\right)-L_{\partial S_{l}}\left(\delta s_{l}\right)=\mathcal{R}_{e}\{-i \omega( & -\int_{\partial_{w^{d}} S_{l}} \delta K_{l}\left(w_{l}-w_{l}^{d}\right)^{*} d L \\
& +\int_{\partial_{w_{\underline{n}}^{d}} S_{l}} \delta M_{l}\left(w_{l, \underline{n}_{l}}-w_{l, \underline{n}_{l}}^{d}\right)^{*} d L \\
& +\int_{\partial_{M_{d}} S_{l}}\left(M_{l}-M_{l}^{d}\right) \delta w_{l, \underline{n}_{l}}^{*} d L \\
& \left.\left.-\int_{\partial_{K^{d}} S_{l}}\left(K_{l}-K_{l}^{d}\right) \delta w_{l}^{*} d L\right)\right\}
\end{aligned}
$$

and with the prescribed interface quantities on $\Gamma$ :

$$
C_{\Gamma}=\mathcal{R}_{e}\left\{-i \omega\left(\int_{\Gamma_{12}}\left(M_{l}-\overline{\bar{M}}_{l}\right) \delta w_{l, \underline{n}_{l}}^{*} d L\right)\right\}
$$

$\mathcal{R}_{e}(Q)$ and $Q^{*}$ designate the real part of $Q$ and the conjugate of $Q$ respectively. Let us note that the terms which appear in this formulation are powers. The use of $\mathcal{R}_{e}(-i \omega)$ is not reducing, because it is redundant with the equation that would result from $\mathcal{I}_{m}(-i \omega)$. In practice, the VTCR procedure consists of two stages: first, one builds admissible fields of $S_{a d}^{l}$ as will be described further on; then, one introduces the boundary conditions through the discretization of the variational formulation associated with a set of admissible fields chosen among the elements of $\mathcal{S}_{a d}^{l}$. Consequently one can make a parallel with Trefftz methods (Kita \& Kamiyar 1995), which also use frontier equations formulations in which fields defined on each substructure are implemented. 
Thus, one obtains the substructure quantity $w_{l, \underline{n}_{l}}$, which must be equal to the interface quantity $\overline{\bar{w}}_{l, \underline{n}_{l}}$. The expression of the interface/substructure $l$ compatibility can then be summarized by the admissible space $\mathcal{S}_{a d}^{\Gamma, l}$ :

$$
\overline{\bar{s}}_{l}=\left(\overline{\bar{w}}_{l, \underline{n}_{l}}, \overline{\bar{M}}_{l}\right) \in \mathcal{S}_{a d}^{\Gamma, l} \quad \Leftrightarrow \quad \overline{\bar{w}}_{l, \underline{n}_{l}}=f_{l}\left(\overline{\bar{M}}_{l}\right)+g_{l}^{d}
$$

where $g_{l}^{d}$ comes from the prescribed quantities on $\partial S_{l}$, and $f_{l}$ is an operator coming from the condensation onto $\Gamma$.

\subsubsection{The final problem}

Once the substructure's equations have been obtained through the substructure/interface compatibility problem (7), the final step consists in solving the equations of the joints by minimizing a residual called the interface error. For example, for a perfectly rigid joint, the interface's behavior is described by the following equations:

$$
\begin{array}{ll}
\text { - compatibility } & \overline{\bar{w}}_{1, \underline{n}_{1}}=\overline{\bar{w}}_{2, \underline{n}_{2}} \\
\text { - equilibrium } & \overline{\bar{M}}_{1}+\overline{\bar{M}}_{2}=0
\end{array}
$$

which is the key to the construction of the interface's $L^{2}$ residual:

$$
E_{\text {Interface }}^{2}=\int_{\Gamma_{12}}\left\|\overline{\bar{w}}_{1, \underline{n}_{1}}-\overline{\bar{w}}_{2, \underline{n}_{2}}\right\|^{2} d L+g \int_{\Gamma_{12}}\left\|\overline{\bar{M}}_{1}+\overline{\bar{M}}_{2}\right\|^{2} d L
$$

where $g$ must homogenizes the dimensions of the two terms. It is actually chosen in order to prevent numerical difficulties relative to the conditioning number of the problem, which are generally encountered with Trefftz methods.

Then, the final problem consists in a constrained minimization:

Final Problem.

$$
\begin{gathered}
\text { Find } \quad \overline{\bar{s}}_{l}=\left(\overline{\bar{w}}_{l, \underline{n}_{l}}, \overline{\bar{M}}_{l}\right) \in \mathcal{S}_{a d}^{\Gamma, l}, \quad \forall l, \quad \text { which minimizes: } \\
E_{\text {Interface }}^{2}=\int_{\Gamma_{12}}\left\|\overline{\bar{w}}_{1, \underline{n}_{1}}-\overline{\bar{w}}_{2, \underline{n}_{2}}\right\|^{2} d L+g \int_{\Gamma_{12}}\left\|\overline{\bar{M}}_{1}+\overline{\bar{M}}_{2}\right\|^{2} d L
\end{gathered}
$$

One can show that this problem is equivalent to the reference problem. As will be detailed later, once the compatibility space $\mathcal{S}_{a d}^{\Gamma, l}$ has been discretized, this constrained minimization problem is solved using Lagrange multipliers.

Let us note that since the interfaces are treated only in a weak way and priority is given to the equations of the substructures it is important to verify that this formulation actually enable us to take into account elaborate joint models. An example will show that this is indeed the case.

\subsection{Construction of the admissible fields}

Let us now describe how the VTCR shape functions are defined. This stage is a fundamental and classical aspect of the method (Ladevèze et al. 2001, Rouch 2003). The VTCR uses a 
two-scale representation of subsets of $\mathcal{S}_{a d}^{l}$. For example, in the vicinity of a point $\underline{X}$ of $S_{l}$, the solution is described as the superposition of modes which can be expressed as follows:

$$
W_{l(\underline{X}, \underline{P})}=e^{i \sqrt{\omega} \underline{P} \cdot \underline{X}} U_{\left.l_{(\underline{X}}, \underline{P}\right)}
$$

The moments are deduced from the constitutive relation:

$$
\mathbf{M}_{l(\underline{X}, \underline{P})}=\left(1+i \eta_{l}\right) \mathbf{K}_{P S} \mathcal{X}\left(W_{l(\underline{X}, \underline{P})}\right)
$$

$e^{i \sqrt{\omega} \underline{P} \cdot \underline{X}}$ represents the "fast" part of the field, which is defined explicitly. $U_{(X, P)}$ is the "slow" part. $\underline{P}$ denotes a vector which characterizes the local vibration mode and is chosen such that the mode verifies the dynamic equations and the constitutive relation of Plate $l$. There are many possible options and choosing among them depends on the zone one is particularly interested in. The VTCR distinguishes the interior zone, the edge zone and the corner zone. For example, let us concentrate on the properties of $n^{\text {th }}$-order complex rays for the interior zone. In this case, $U_{l}$ includes an $n^{t h}$-order polynomial in $\underline{X}$. If the damping factor $\eta_{l}$ is small, $U_{l}$ can be expressed as:

$$
U_{l(\underline{X}, \underline{P})}=e^{\frac{\eta_{l}}{4} \sqrt{\omega} \underline{P} \cdot \underline{X}} \mathbf{V}_{(\underline{X}, \underline{P})}\left[\underline{a}_{l(\underline{P})}\right]
$$

The coefficients $\underline{a}_{l(\underline{P})}$ of the polynomial $\mathbf{V}_{(\underline{X}, \underline{P})}\left[\underline{a}_{l(\underline{P})}\right]$ are unknowns; they form a column $\left[\underline{a}_{l(\underline{P})}\right]$ of "generalized amplitudes". Consequently, all the unknowns are large-wavelength quantities. The damping of the wave being modeled is represented by the expression $e^{\frac{\eta_{l}}{4} \sqrt{\omega} \underline{P} \cdot \underline{X} .}$.

These fields can be viewed as vibration modes in an infinite domain having the same mechanical properties as Substructure $S_{l}$ : therefore, they belong to $\mathcal{S}_{a d}^{l}$.

In the following sections, for the sake of simplicity, we will consider the particular case of a $0^{\text {th }}$-order complex ray and omit the index $l$ of the plate. In that case, the simplified expression of the complex ray is:

$$
W_{(\underline{X}, \underline{P})}=a_{(\underline{P})} e^{\left(\frac{\eta}{4} \sqrt{\omega} \underline{P} \cdot \underline{X}\right)} e^{(i \sqrt{\omega} \underline{P} \cdot \underline{X})}=a_{(\underline{P})} w_{e}(\underline{X}, \underline{P})
$$

$a_{(\underline{P})}$ being the complex magnitude of the elementary complex ray $w_{e(\underline{X}, \underline{P})}$.

The admissibility relation

$$
\Delta \Delta W-k^{4} W=0 \quad \text { on } S
$$

where $\quad k^{4}=\frac{3 \rho \omega^{2}\left(1-\nu^{2}\right)}{(1+i \eta) E h^{2}}$

requires:

$$
(\underline{P} . \underline{P})^{2}=r^{4} \quad \text { with } \quad r^{4}=\frac{3 \rho\left(1-\nu^{2}\right)}{E h^{2}}
$$

Therefore, the locus of the end of the admissible wave vector $\underline{P}$ for the interior zone is a circle $\mathcal{C}$ of radius $r$ (see Fig. 3). This curve is a characteristic of the material. As one follows the circular path, every direction of the plate is taken into account. Examples of such modes can be seen in Fig. 4. The definitions of corner modes and edge modes are very similar. 


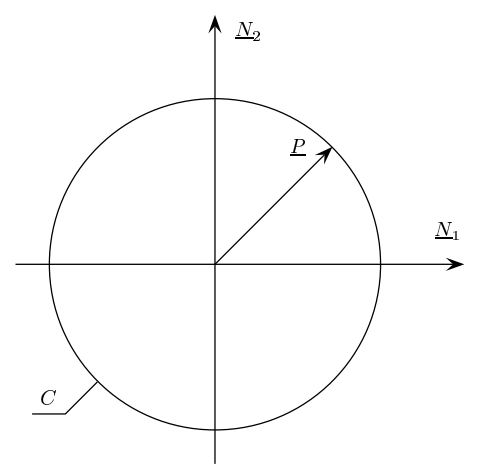

Fig. 3. Admissible $\underline{P}$ for interior modes
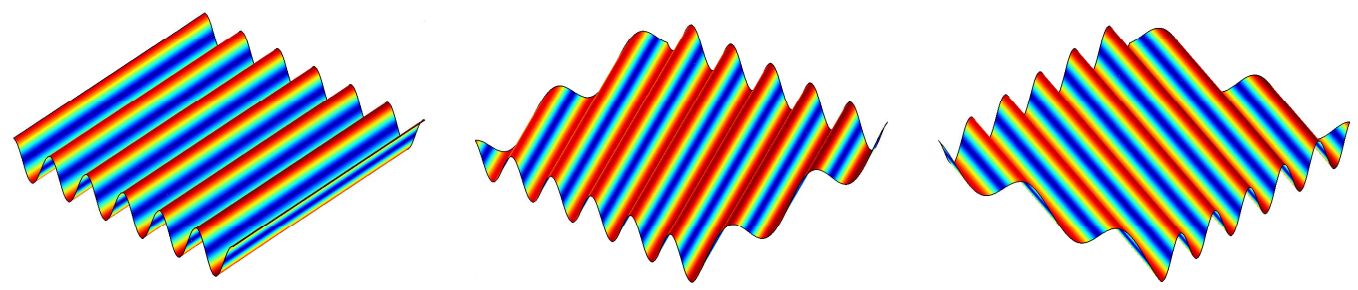

Fig. 4. Admissible interior modes for a plate

For the interior zone, one takes into account all admissible ray directions. The solution $w^{\text {sol }}$ can be expressed as:

$$
w_{(\underline{X})}^{s o l}=\int_{\mathcal{C}} W_{(\underline{X}, \underline{P})} d s
$$

The integrals are calculated along Curve $\mathcal{C}$. Clearly, $w^{\text {sol }}$ depends on the generalized amplitude $[\underline{a}]$ relative to $\underline{P}$ defined on $\mathcal{C}$.

\subsection{The discretized form of the VTCR}

All that is needed in order to derive approximations from the VTCR is the selection of a subspace $\mathcal{S}_{a d, h}^{l}$ of $\mathcal{S}_{a d}^{l}$. In practice, one discretizes Curve $\mathcal{C}$ into $n$ elements which can be of different sizes (for example, in Fig. $5, \mathcal{C}$ is discretized into eight elements of the same size.) The amplitude $a_{(\underline{P})}$ is considered to be constant throughout angular sectors $a_{i}^{h}$. Then:

$$
w_{(\underline{X})}^{h}=\sum_{i=1}^{n} a_{i}^{h} \int_{\mathcal{C}_{i}} w_{e}(\underline{X}, \underline{P}) d s=\sum_{i=1}^{n} a_{i}^{h} w_{i(\underline{X})}^{h}=\underline{W}_{(\underline{X})}^{h} \cdot \underline{a}^{h}
$$

The generalized amplitude $a_{i}^{h}$ is associated with the basic function $w_{i(\underline{X})}^{h}$ corresponding to the integral of an elementary ray over an angular sector related to $\mathcal{C}_{i}$. Let us note that since the admissible Equations (1) are linear, the basic function $w_{i(\underline{X})}^{h}$ derive from the integration 
in respect with the ray direction $\varphi$ and keep on satisfying the governing equations. This point preserves the variational consistency of the VTCR approach. In practice, the integration is performed thanks to a multiscale approach : indeed, a complex rays is composed of two parts : a fast one and a slow one. The fast part of the complex ray is treated analytically while the slow part is integrated by a numerical approach.

Then, the subspace $\mathcal{S}_{a d, h}^{l}$ of the approximations deduced from $\mathcal{S}_{a d}^{l}$ is defined by:

$$
s_{l}^{h} \in \mathcal{S}_{a d, h}^{l} \quad \Leftrightarrow \quad\left\{\begin{array}{l}
w_{(\underline{X})}^{h}=\underline{W_{(\underline{X})}^{h}} \cdot \underline{a}^{h} \\
M_{(\underline{X})}^{h}=\underline{\Lambda}_{(\underline{X})}^{h} \cdot \underline{a}^{h}
\end{array}\right.
$$

The same procedure can be applied to corner zones and edge zones.

In order to end up with a finite-dimension problem, the interface quantities must also be discretized. Let us note that there is no obligation for the interface quantities and the substructure quantities to be discretized in the same way.

$$
\overline{\bar{s}}_{l}^{h} \in \mathcal{S}_{a d, h}^{\Gamma, l} \quad \Leftrightarrow \quad\left\{\begin{array}{l}
\overline{\bar{w}}_{(\underline{X})}^{h}=\underline{\bar{W}}_{(\underline{X})}^{h} \cdot \underline{\overline{\bar{a}}}^{h} \\
\overline{\bar{M}}_{(\underline{X})}^{h}=\underline{\bar{\Lambda}}_{(\underline{X})}^{h} \cdot \underline{\bar{a}}^{h}
\end{array}\right.
$$

The interface/substructure compatibility problem (7) leads to the following linear problem:

$$
\left(\underline{\overline{\bar{a}}}_{l}, \underline{a}_{l}\right) \in \mathcal{S}_{a d, h}^{\Gamma, l}, \quad \forall l, \quad \Leftrightarrow \quad\left[\mathbb{K}_{a d m}\right]\left[\frac{\underline{a}}{\overline{\bar{a}}}\right]=\underline{F}_{a d m}
$$

while the interface error leads to a quadratic form:

$$
E_{\text {Interface }}^{2}=\frac{1}{2}^{t}[\underline{\underline{\bar{a}}}]\left[\mathbb{K}_{\text {err }}\right]\left[\frac{\underline{a}}{\overline{\bar{a}}}\right]
$$

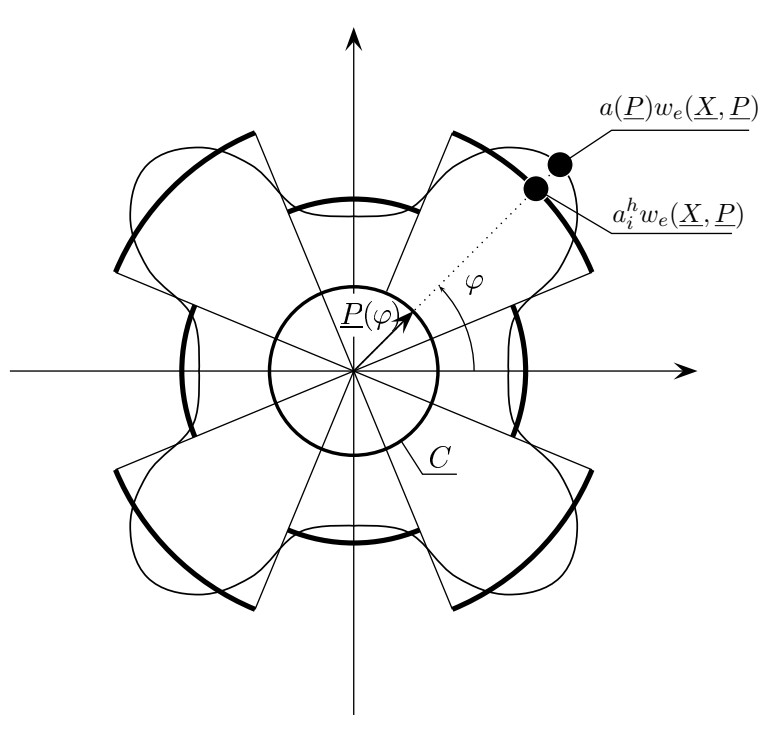

Fig. 5. Admissible interior modes for a plate 
Thus, the final problem (10) can be expressed as the following constrained minimization problem:

Final Discretized Problem.

$$
\begin{gathered}
\text { Find }(\underline{a}, \underline{\bar{a}}) \text { which minimizes } E_{\text {Interface }}^{2}=\frac{1}{2}^{t}\left[\frac{a}{\overline{\bar{a}}}\right]\left[\mathbb{K}_{\text {err }}\right]\left[\frac{a}{\overline{\bar{a}}}\right] \\
\text { under the compatibility constraints }\left[\mathbb{K}_{a d m}\right]\left[\frac{a}{\underline{\bar{a}}}\right]=\underline{F}_{a d m}
\end{gathered}
$$

Finally, the constrained minimization problem is solved using Lagrange multipliers $\underline{\lambda}$ :

$$
\left[\begin{array}{cc}
\frac{1}{2}\left(\mathbb{K}_{\text {err }}+{ }^{t} \mathbb{K}_{\text {err }}\right) & -{ }^{t} \mathbb{K}_{a d m} \\
-\mathbb{K}_{a d m} & 0
\end{array}\right]\left[\begin{array}{c}
{\left[\begin{array}{c}
\underline{a} \\
\underline{\bar{a}}
\end{array}\right]} \\
\underline{\lambda}
\end{array}\right]=\left[\begin{array}{c}
\underline{0} \\
\underline{F}_{a d m}
\end{array}\right]
$$

Let us note that the minimization constraints do not depend on the joint parameters, which are contained in the residual: the admissibility system is built once and for all, and changes in interface behavior affect only the residual matrix $\mathbb{K}_{e r r}$.

Anterior studies have shown that in practice, it is not necessary to take into account order greater than zero in the slow part $\underline{a}^{h}$, which will be the case in the examples presented in this paper. In the rest of the presentation, for numerical reasons, we choose to discretize the interface quantities using the same space $S_{a d, h}^{l}$ as for the corresponding substructure quantities, or a subspace of this space. The discretization of the interface quantities will be studied in the last part.

\subsection{Effective quantities}

From a mechanical point of view, the spatial distribution of the solution has no reliable physical meaning in the medium-frequency range.

Indeed, the response at a specific location and for a specific frequency is extremely sensitive to perturbations. In order to interpret the response, spatially-averaged quantities are more useful. Thus, it is necessary to extract from the solution some effective quantities defined on a domain greater than one wavelength.

On a given substructure $S$, let us designate the quantities of interest by $Q_{(\underline{X})}$, which can represent, for example, the strain energy, the kinetic energy or the dissipation work. In the vicinity of any test point, the associated effective quantities are defined over a domain $D$ by:

$$
Q_{D}=\frac{1}{D} \int_{D}\left|Q_{(\underline{X})}\right| d \underline{X}
$$

For instance, in the last section, the domains $D$ considered will be composed of each plate $S_{i}$ of the structure. 


\section{Modeling of the interfaces}

Today, the modeling of beams, plates, shells and their combinations no longer presents difficulties for the VTCR. Dedicated techniques allows us to implement more precise details like uncertainties (holes for an example) located in the interior of substructures (Ladevèze, et al. 2003). However in actual thin structures (such as cars, satellites or launchers), most of the damping, which governs the amplitude of the response to dynamic solicitations, is localized in the different connections between substructures. These joints (whether welded, riveted or bolted) modify the structural response considerably and can be used by engineers to adjust the structure's vibrational properties. As an illustration, experiments on three generic assemblies (a flat plate, a folded plate and a welded plate) clearly show that joint stiffness and damping must be taken into account. The experimental setup is not described in this paper since it is the subject of an additional one. The results are synthesized in Table I and on Fig. 6. They shows that the damping of the joints depends on the type of connexion which is used, and on the frequency of the solicitation. Consequently the modeling of the joints must be improved in order to take into account more realistic joint behavior.

\begin{tabular}{|c|c|c|c|c|}
\hline Frequency $(\mathrm{Hz})$ & $200-400$ & $400-800$ & $800-1600$ & $1600-3200$ \\
\hline \multicolumn{5}{|c|}{ Flat plate } \\
\hline$P_{i n j}\left(10^{-6} W\right)$ & 4.61 & 3.90 & 1.38 & 0.93 \\
\hline$V^{2}\left(10^{-6} m^{2} \cdot s^{-2}\right)$ & 13.48 & 5.17 & 1.42 & 0.41 \\
\hline$\eta$ & 0.000467 & 0.000513 & 0.000332 & 0.000389 \\
\hline$\Delta \eta$ & 0.000173 & 0.000496 & 0.000001 & 0.000005 \\
\hline \multicolumn{5}{|c|}{ Folded plate } \\
\hline$P_{i n j}\left(10^{-6} W\right)$ & 0.97 & 1.01 & 0.48 & 0.28 \\
\hline$V_{1}^{2}\left(10^{-6} m^{2} . s^{-2}\right)$ & 1.56 & 0.89 & 0.19 & 0.05 \\
\hline$V_{2}^{2}\left(10^{-6} m^{2} \cdot s^{-2}\right)$ & 2.04 & 1.39 & 0.44 & 0.37 \\
\hline$\eta$ & 0.000376 & 0.000311 & 0.000302 & 0.000267 \\
\hline$\Delta \eta$ & 0.000002 & 0.000003 & 0.000006 & 0.000003 \\
\hline \multicolumn{5}{|c|}{ Welded plate } \\
\hline$P_{i n j}\left(10^{-6} W\right)$ & 6.44 & 1.93 & 0.92 & 0.10 \\
\hline$V_{1}^{2}\left(10^{-6} m^{2} \cdot s^{-2}\right)$ & 0.94 & 0.18 & 0.10 & 0.03 \\
\hline$V_{2}^{2}\left(10^{-6} m^{2} \cdot s^{-2}\right)$ & 1.50 & 0.67 & 0.19 & 0.03 \\
\hline$\eta$ & 0.003795 & 0.002398 & 0.001148 & 0.000269 \\
\hline$\Delta \eta$ & 0.000075 & 0.000327 & 0.000217 & 0.000138 \\
\hline
\end{tabular}

Table I. Results of global damping estimation

The joints presented here are described by distributed interface parameters, such as mass, stiffness and damping coefficients (see Fig. 7), which enable us to model complex heterogeneous connections. These coefficients can be identified on generic assemblies to 


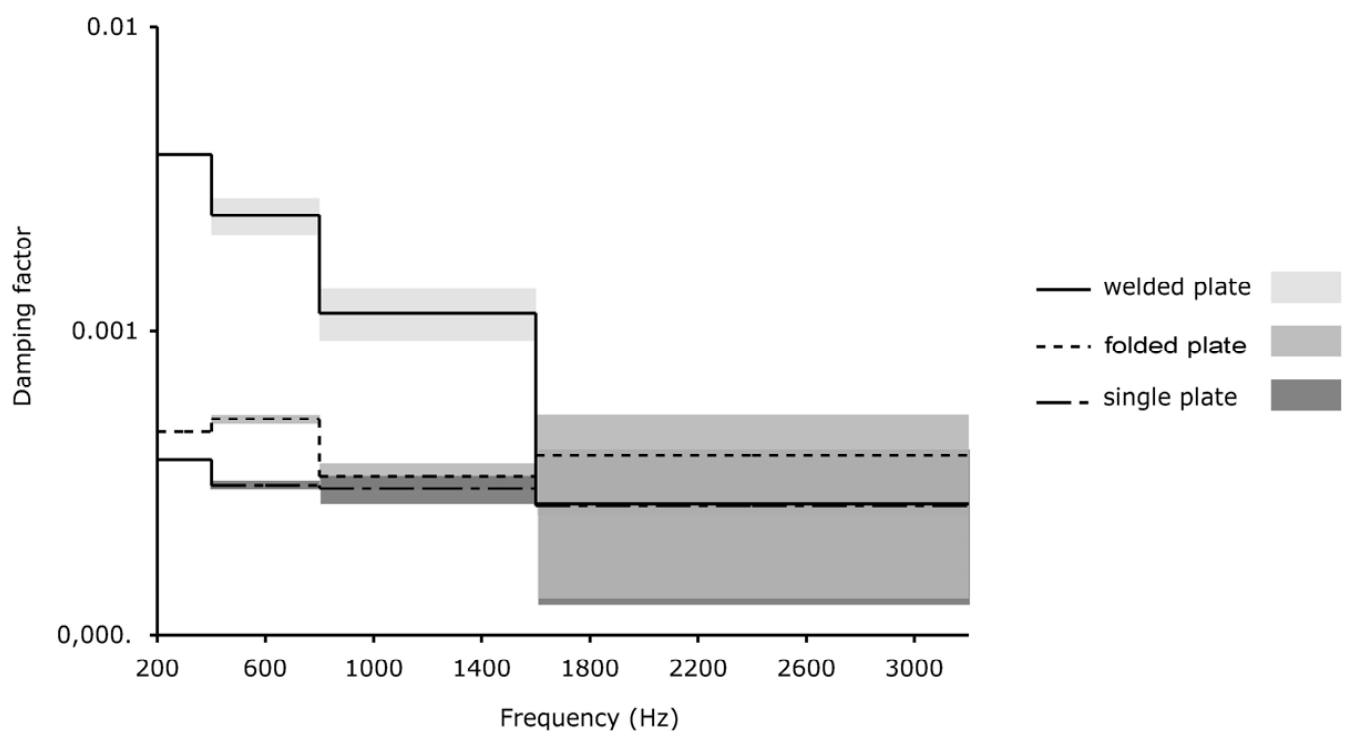

Fig. 6. Global damping values for several frequency ranges

provide more realistic structural behavior.

\subsection{Modeling of the joints}

The local behavior can be modeled by interface relations which represent elastic and/or dissipative joint characteristics. Each joint is considered to be a mechanical entity whose own specific behavior depends on the type of connection. Many different types of joints can be modeled by this approach.

As in the case of perfect joints, there are four local boundary/interface conditions for each side of a plate: two equilibrium equations (one for the bending moments and the other
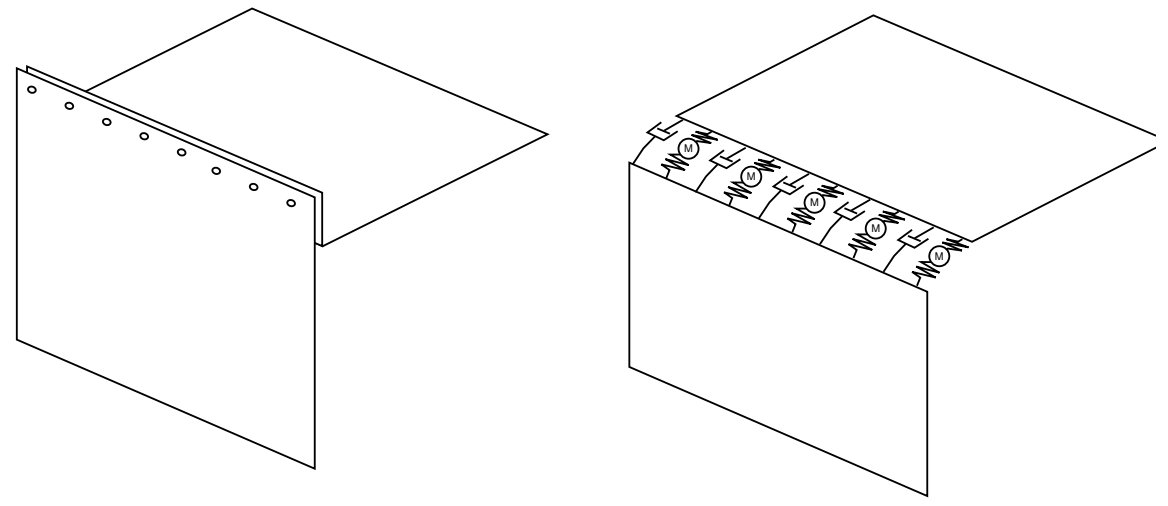

Fig. 7. Generic assembly modeling 
for the Kirchhoff shears) and two compatibility equations (one for the displacements and the other for the slopes). For example, Equation (9) is the residual of perfectly rigid interface equations for bending moments:

$$
\left\{\begin{aligned}
w_{l, \underline{n}_{l}}-w_{m, \underline{n}_{m}} & =0 & & \text { continuity of the slopes } \\
M_{l}+M_{m} & =0 & & \text { equilibrium of the bending moments }
\end{aligned}\right.
$$

The residual for Kirchhoff shears would be very similar. In order to obtain a more realistic model of the connections, let us now describe the local behavior by the following equations of a complex elastic and/or dissipative joint:

$$
\left\{\begin{aligned}
\frac{1}{2}\left(-M_{l}+M_{m}\right) & =-k_{b}\left(1+i \eta_{b}\right)\left(w_{l, \underline{n}_{l}}-w_{m, \underline{n}_{m}}\right) & & \text { constitutive relation } \\
M_{l}+M_{m} & =-\frac{J \omega^{2}}{2}\left(w_{l, \underline{n}_{l}}+w_{m, \underline{n}_{m}}\right) & & \text { dynamic equation }
\end{aligned}\right.
$$

where $k_{b}$ and $J$ are the line density of bending stiffness and inertia respectively, and $\eta_{b}$ models the corresponding dissipative part.

We built the residual of Equations (27) as:

$$
\begin{aligned}
E_{\text {Interface }}^{2} & =\int_{\Gamma_{12}}\left\|\frac{1}{2}\left(-M_{l}+M_{m}\right)+k_{b}\left(1+i \eta_{b}\right)\left(w_{l, \underline{n}_{l}}-w_{m, \underline{n}_{m}}\right)\right\|^{2} d L \\
& +g \int_{\Gamma_{12}}\left\|M_{l}+M_{m}+\frac{J \omega^{2}}{2}\left(w_{l, \underline{n}_{l}}+w_{m, \underline{n}_{m}}\right)\right\|^{2} d L
\end{aligned}
$$

where $g$ is chosen to balance the order of magnitude of the two terms in order to avoid numerical problems.

Similarly, we can introduce new clamping conditions between the structure and the ground, which seems to be more realistic than prescribing zero slope:

$$
M_{l}=-k_{b}\left(1+i \eta_{b}\right) w_{l, \underline{n}_{l}} \quad \text { bending spring with the ground }
$$

This condition can be implemented thanks to the following residual:

$$
E_{\text {Interface }}^{2}=\int_{\Gamma_{0}}\left\|M_{l}+k_{b}\left(1+i \eta_{b}\right) w_{l, \underline{n}_{l}}\right\|^{2} d L
$$

Let us note that in order to improve accuracy the interface coefficients, such as stiffness, mass and damping, can be made to vary along the interface. For example, a riveted joint can be modeled by a stiffness coefficient which is infinite and zero alternatively, as shown in Fig. 8 (where the joint's stiffness is denoted $k$ ). In order to implement such parameters numerically, it is necessary to discretize them. Considering that in actual structures most of the joints have periodic characteristics, we chose to use a Fourier decomposition of the interface coefficients, such as:

$$
k_{(\underline{X})}=\sum_{n=-\infty}^{+\infty} k_{n} e^{i n \underline{\lambda} \cdot \underline{X}} \simeq \sum_{n=-N}^{+N} k_{n} e^{i n \underline{\lambda} \cdot \underline{X}} \quad \underline{X} \in \Gamma
$$

with $\underline{\lambda}$ being the period of the pattern describing the junction.

Fig. 8 and Fig. 9 show an example of such a calculation in which the joint's bending stiffness and translation stiffness have both been taken into account. The VTCR calculations 

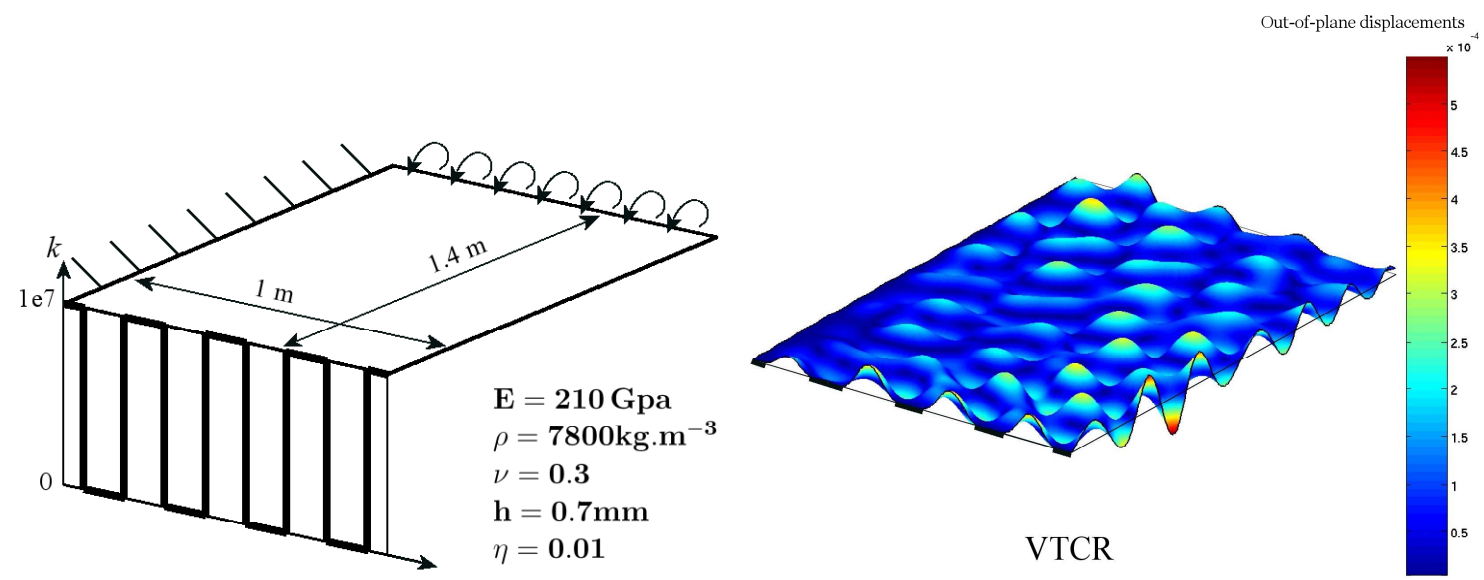

Fig. 8. The heterogeneous interface model and its calculation

required 260 degrees of freedom (128 interior modes and 33 edge modes per edge). The results confirm that the boundary conditions are accurately respected. In this case, modeling the joint with constant parameters along the interface could not have given an acceptable result. In order to validate this VTCR solution, we compared it with the solution obtained with the traditional FEM code NASTRAN. The FEM calculation was conducted using at least 10 elements per wavelength, according to the so-called "rule of the thumb" (see (Ihlenburg \& Babuska 1995), (Ihlenburg \& Babuska 1997)). 82, 863 degrees of freedom were required. The maximum displacements obtained were $5.2610^{-4} \mathrm{~m}$ with the VTCR and $4.9610^{-4} \mathrm{~m}$ with NASTRAN, which amounts to a difference of about $6 \%$.

\subsection{Numerical example}

In this section, we present the influence of the joint's bending stiffness and damping on the response of an assembly of two plates connected by a complex joint (see Fig. 10). Here, the interface parameters were considered to be constant along the interface. To make comparisons possible, we used the effective displacements of each plate as defined in $\mathbf{2 . 5}$. The domains $D$ considered in this definition are composed of each plate $S_{i}$ of the structure. These quantities were averaged over $[100 \mathrm{~Hz}, 200 \mathrm{~Hz}]$. Let us note that for the calculations with the VTCR only 200 degrees of freedom were used: 68 d.o.f. for a plate (32 interior modes and 9 edge modes per edge), and 32 other d.o.f. for the interface quantities relative to the plate. The minimization contraints add 68 Lagrange multipliers per plate (as many as the number of test functions of each plate). Consequently, the final size of the problems is 336 degrees of freedom, which seems to be very reasonable.

We can observe on Fig. 11 that a stiffness per unit length greater than $10^{3}$ N.m.rd $d^{-1}$ is equivalent to a perfectly rigid joint. Similarly, for a joint stiffness equal to $10^{2}$ N.m.r. $d^{-1}$, damping is significant if it is greater than $10^{-1}$. This value can seem very high, but it should not be compared directly to the damping of the plate (equal to 0.01 ) because what matters 


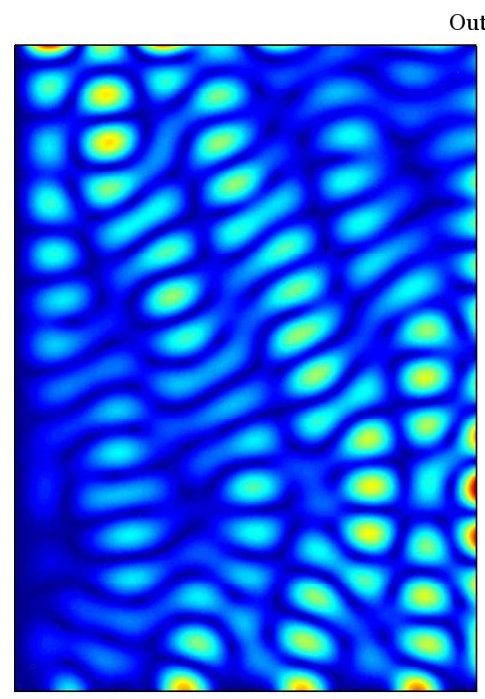

VTCR

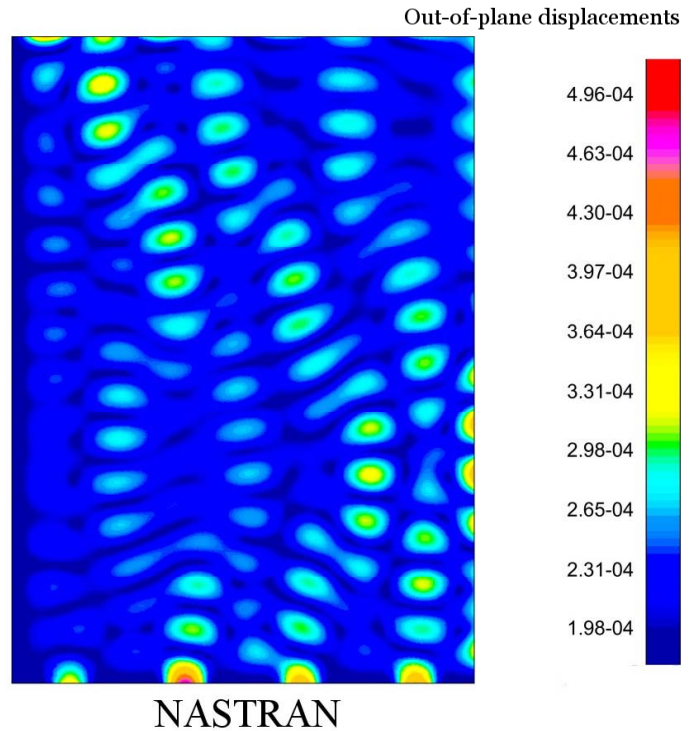

Fig. 9. Heterogeneous interface $(245 \mathrm{~Hz})$ : (a) VTCR solution, (b) NASTRAN solution

is the contribution of the joint's dissipation to the total energy dissipation of the structure.

\subsection{Example}

Let us compare the results obtained with the classical VTCR formulation, used as the reference, with those obtained by the new formulation using the minimization of the interface's residual. Here the discretization on each plate required 68 degrees of freedom for the classical VTCR formulation (32 interior basis functions per plate, 9 edge modes per edge), and the new formulation introduces for each plate 32 interface degrees of freedom and 68 Lagrange

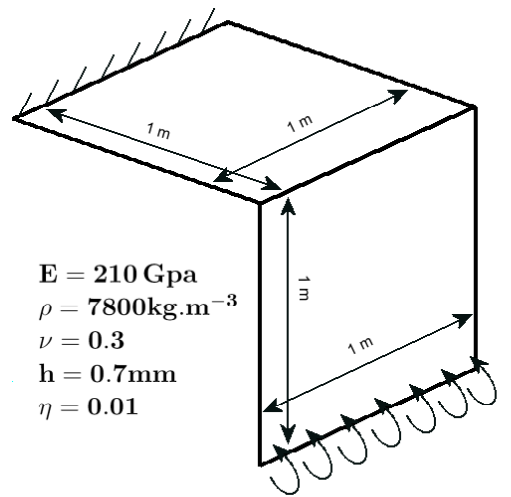

Fig. 10. Assembly of two plates with a complex constant joint 

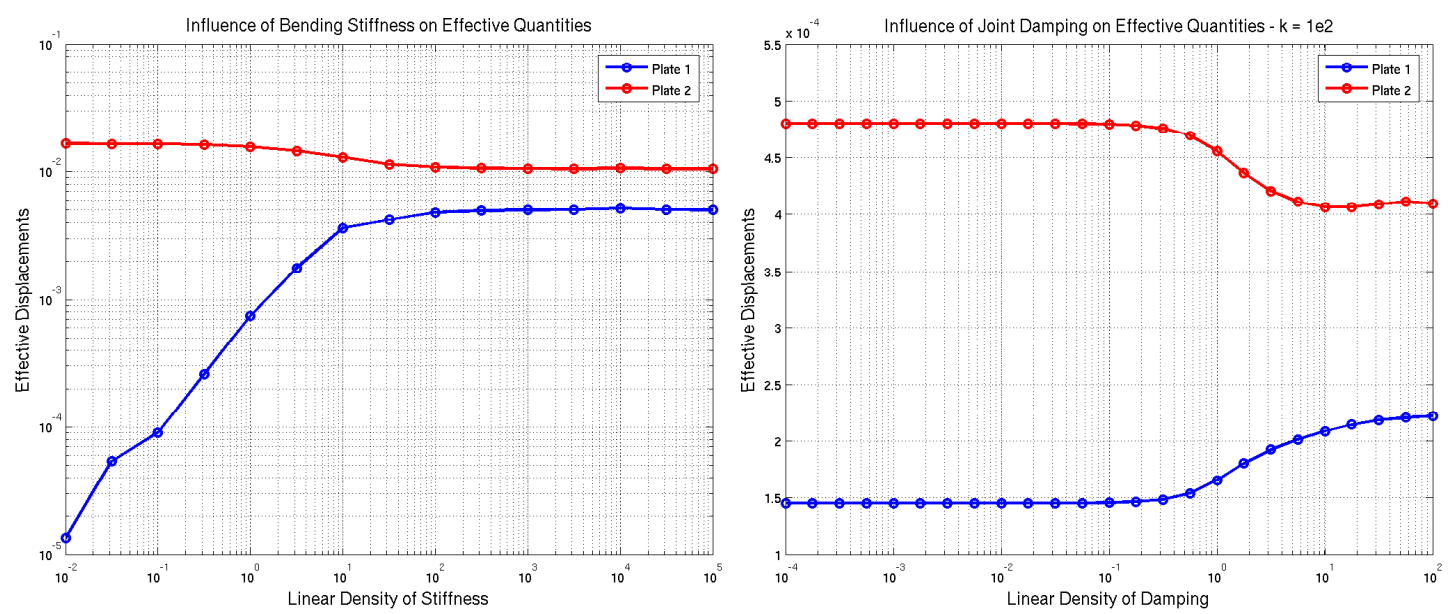

Fig. 11. Influence of the joint's parameters: (a) stiffness (zero damping), (b) damping (stiffness equal to $\left.10^{2} N \cdot m \cdot r d^{-1}\right)$

multipliers respectively. Fig. 12 shows the solutions obtained by the two methods for the two-plate assembly with a perfectly rigid joint and a $220 \mathrm{~Hz}$ solicitation. The solutions are very similar, as can be seen from the effective displacements (Table II).

\begin{tabular}{|c|c|c|}
\cline { 2 - 3 } \multicolumn{1}{c|}{} & \multicolumn{2}{c|}{ Effective quantities } \\
\cline { 2 - 3 } \multicolumn{1}{c|}{} & Classical formulation & Substructured formulation \\
\hline Plate 1 & $16.33310^{5}$ & $16.38910^{5}$ \\
\hline Plate 2 & $12.11710^{5}$ & $12.06110^{5}$ \\
\hline
\end{tabular}

Table II. Validation of the substructured version of the VTCR

The next calculations concern the same assembly of two plates, solicited at $100 \mathrm{~Hz}$, but with a complex joint and different values of the joint's stiffness. The discretization requires 68 degrees of freedom per plate (32 interior modes and 9 edge modes per edge) for each substructures. Different discretizations were used for the interface quantities: discretizations (a), (b) and (c) consisted of the border complex rays (9 edge modes per edge), the interior complex rays (32 interior modes per plate), and both the border and the interior complex rays (32 interior modes and 9 edge modes per edge) respectively. (d) is the reference calculated with the classical global formulation involving 68 degrees of freedom per plate (32 interior modes and 9 edge modes per edge).

Fig. 13 shows that the results are exactly the same and that a coarse discretization of the interface is sufficient, provided that it contains the interface's complex rays relative to the joint being considered. 


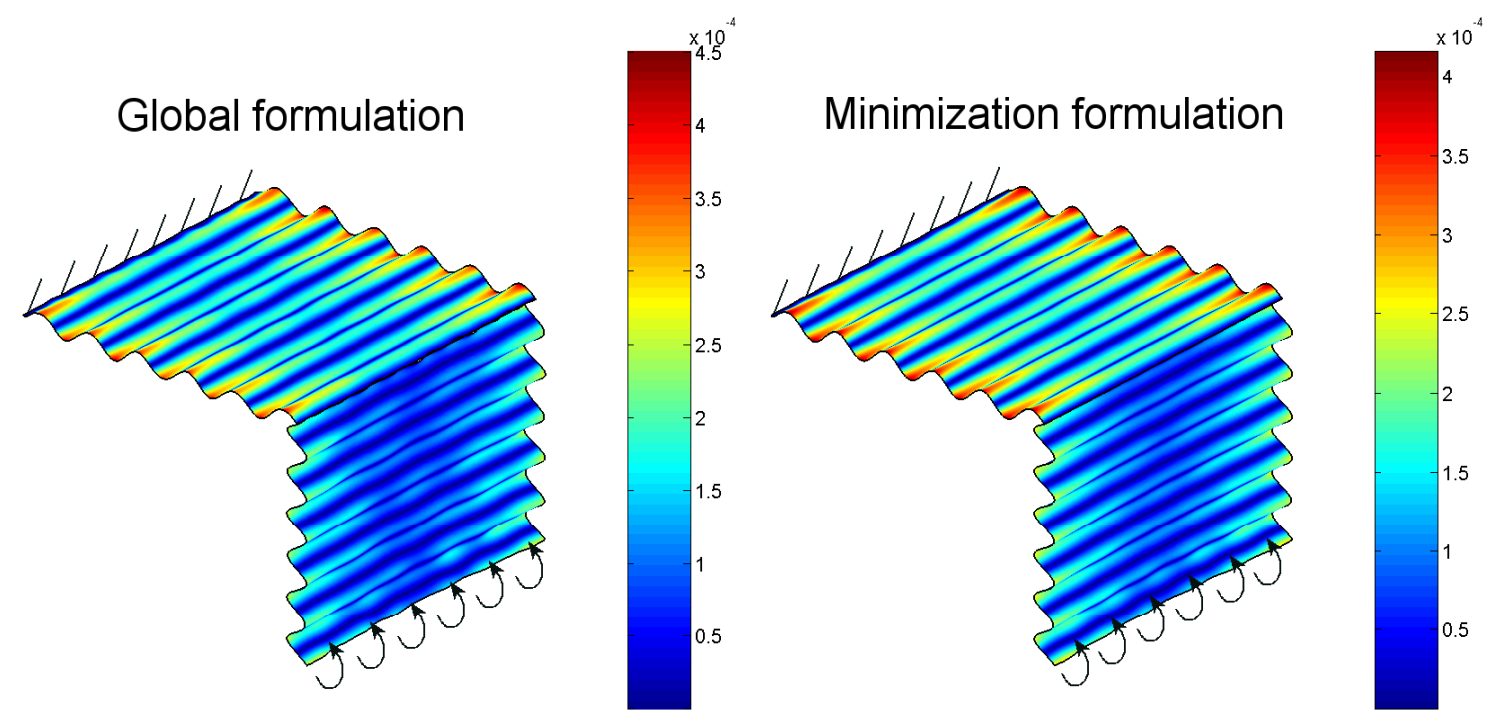

Fig. 12. Solutions obtained with the two formulations

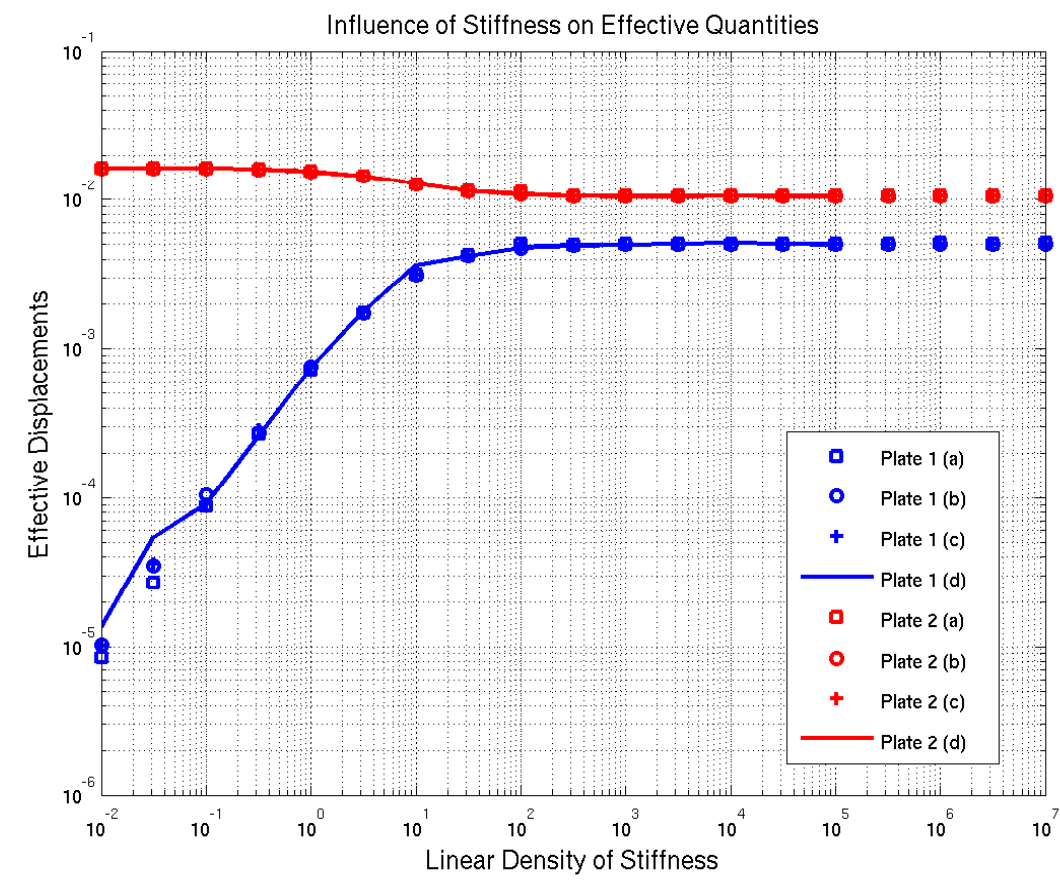

Fig. 13. Comparison between the classical VTCR and the substructured VTCR

\section{Conclusion}

The Variational Theory of Complex Rays is a predictive approach which is very well-suited to medium-frequency vibration analysis of complex structures. Its main features are the 
use of shape functions with a strong mechanical meaning and a variational formulation which results in a very low cost, independent of the frequency. The variational formulation is associated with the boundary conditions, which is very useful in order to take into account different complex connections between substructures. Since the modeling of actual heterogeneous joints is very important in order to obtain an accurate response in mediumfrequency vibrations with this type of structure, we introduced more sophisticated interface equations through mass-stiffness-damping joint behavior. We presented a new formulation of the theory which enables one to analyze the same structure with different connections more conveniently. This new formulation is based on the minimization of a $L^{2}$ residual of interface equations while the admissibility of the well-modeled substructures is enforced one and for all by minimization constraints. This formulation should not meet any theoretical difficulty to treat more complex structures like assemblies of beams, plates, or shells. In future works, we will take advantage of this formulation to identify the parameters of the joints using experimental results.

\section{References}

R. Craig \& M. Bampton (1968). 'Coupling of Substructures for Dynamic Analysis'. AIAA Journal 6(7):1313-1319.

A. Deraemaeker, et al. (1999). 'Dispersion and pollution of the FEM solution for the Helmholtz equation in one, two and three dimensions'. Int. J. Numer. Meth. Engng. 46:471-499.

C. Farhat, et al. (1994). 'A Transient FETI Methodology for Large-Scale Parallel Implicit Computations in Structural Mechanics'. International Journal for Numerical Methods in Engineering 37(11):1945-1975.

C. Farhat \& M. Géradin (1994). 'On a Component Mode Synthesis Method and its Application to Incompatible Substructures'. Computers \&5 Structures 51(5):459-473.

C. Farhat, et al. (2001). 'The Discontinuous Enrichment Method'. Comp. Meth. Appl. Mech. Engrg. 190(48):6455-6479.

C. Farhat, et al. (2003). 'A Discontinuous Galerkin Method with Lagrange Multipliers for the Solution of Helmholtz Problems in the Mid-Frequency Regime'. Comput. Methods Appl. Mech. Engrg. 192:1389-1419.

C. Farhat \& J. Mandel (1998). 'The Two-Level FETI Method for Static and Dynamic Plate Problems Part I: An Optimal Iterative Solver for Biharmonic Systems'. Comput. Methods Appl. Mech. Engrg. 155:129-151.

C. Farhat \& F.-X. Roux (1991). 'A Method for Finite Element Tearing and Interconnecting and its Parallel Solution Algorithm'. International Journal for Numerical Methods in Engineering 32(6):1205-1227.

I. Harari \& S. Haham (1998). 'Improved Finite Element Methods for Elastic Waves'. Comp. Meth. Appl. Mech. Engrg. 166(1-2):143-164.

W. C. Hurty (1965). 'Dynamic Analysis of Structural Systems Using Component Modes'. AIAA Journal 37(4):678-685.

F. Ihlenburg \& I. Babuska (1995). 'Finite Element Solution of the Helmholtz Equation with High Wave Number. Part I: The h-Version of the FEM'. Computers \& Mathematics with Applications 30(9):9-37.

F. Ihlenburg \& I. Babuska (1997). 'Finite Element Solution of the Helmholtz Equation with High Wave Number. Part 2: The h-p-Version of the FEM'. Siam J. of Numer. Anal. 34(1):315-358. 
April 10, 2006 9:0

E. Kita \& N. Kamiyar (1995). 'Trefftz methods: an overview'. Advances in Engineering Software 18(89).

P. Ladevèze (1998). Nonlinear Computational Structural Method : New Approaches and NonIncremental Methods of Calculations. Springer-Verlag.

P. Ladevèze, et al. (2001). 'The variational theory of complex rays for the calculation of mediumfrequency vibrations'. Engineering Computations 18(1/2):193-214.

P. Ladevèze, et al. (2003). 'A Multiscale Computational Method for Medium-Frequency Vibrations of Assemblies of Heterogeneous Plates'. Computers \& Structures 81:1267-1276.

P. Ladevèze \& H. Riou (2005). 'Calculation of Medium-Frequency Vibrations over a Wide Frequency Range'. Comp. Meth. Appl. Mech. Engrg. 194(27-29):3167-3191.

R. Lyon \& G. Maidanick (1967). 'Power flow between linearly coupled oscillators'. JASA 34(5):623639.

J. Mandel (1993). 'Balancing Domain Decomposition'. Communications in Applied Numerical Methods 9:233-241.

R. McNeal (1971). 'A Hybrid Method For Component Mode Synthesis'. Computers \& Structures 1(4):581-601.

P. Rouch (2003). Modélisation et Calcul Des Structures Complexes En Moyennes Fréquences Par la Théorie Variationnelle Des Rayons Complexes. Ph.D. thesis, ENS Cachan.

P. Rouch \& P. Ladevèze (2003). "The variational theory of complex rays : a predictive tool for medium-frequency vibrations'. Comp. Meth. Appl. Mech. Engrg. 192:3301-3315.

F.-X. Roux (1990). 'Méthodes de Résolution Par Sous-Domaines En Statique'. La Recharche Aérospatiale 1:37-48. 